
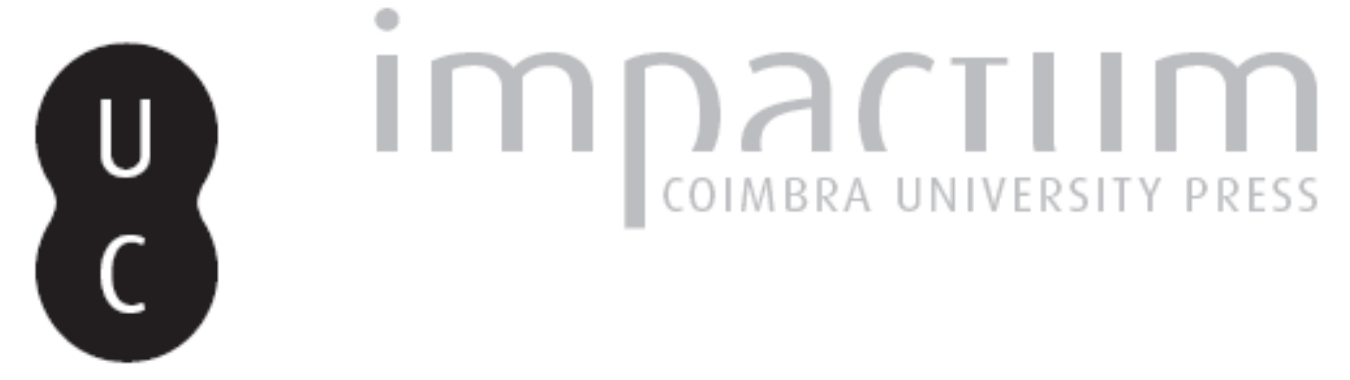

\title{
Avaliação de riscos: comparação entre vários métodos de avaliação de risco de natureza semi-quantitativa
}

Autor(es): $\quad$ Carvalho, Filipa; Melo, Rui Bettencourt

Publicado por: Associação Portuguesa de Riscos, Prevenção e Segurança

URL persistente:

URI:http://hdl.handle.net/10316.2/36047

DOI:

DOI:http://dx.doi.org/10.14195/1647-7723_18_4

Accessed : $\quad$ 26-Apr-2023 15:59:09

A navegação consulta e descarregamento dos títulos inseridos nas Bibliotecas Digitais UC Digitalis, UC Pombalina e UC Impactum, pressupõem a aceitação plena e sem reservas dos Termos e Condições de Uso destas Bibliotecas Digitais, disponíveis em https://digitalis.uc.pt/pt-pt/termos.

Conforme exposto nos referidos Termos e Condições de Uso, o descarregamento de títulos de acesso restrito requer uma licença válida de autorização devendo o utilizador aceder ao(s) documento(s) a partir de um endereço de IP da instituição detentora da supramencionada licença.

Ao utilizador é apenas permitido o descarregamento para uso pessoal, pelo que o emprego do(s) título(s) descarregado(s) para outro fim, designadamente comercial, carece de autorização do respetivo autor ou editor da obra.

Na medida em que todas as obras da UC Digitalis se encontram protegidas pelo Código do Direito de Autor e Direitos Conexos e demais legislação aplicável, toda a cópia, parcial ou total, deste documento, nos casos em que é legalmente admitida, deverá conter ou fazer-se acompanhar por este aviso.

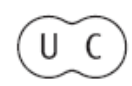




\section{territorium}

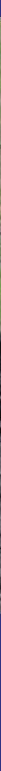

\section{Afirmar as Ciências Cindínicas}

Revista da Associação Portuguesa de Riscos, Prevenção e Segurança 


\section{AVALIAÇÃO DE RISCOS: COMPARAÇÃO ENTRE VÁRIOS MÉTODOS DE AVALIAÇÃO DE RISCO DE NATUREZA} SEMI-QUANTITATIVA*

Filipa Carvalho

Secção Autónoma de Ergonomia, Faculdade de Motricidade Humana da Universidade Técnica de Lisboa, fcarvalho@fmh.utl.pt

Rui Bettencourt Melo

Secção Autónoma de Ergonomia, Faculdade de Motricidade Humana da Universidade Técnica de Lisboa, rmelo@fmh.utl.pt

\section{RESUMO}

Este artigo objectiva dar a conhecer os resultados de um estudo, realizado no âmbito do mestrado em Ergonomia na Segurança no Trabalho, o qual se centrou na análise comparativa entre 10 métodos de avaliação de risco, de natureza semi-quantitativa. Com este estudo, pretendeu-se investigar se o Nível de Risco obtido pelos 10 métodos era idêntico para cada um dos riscos previamente identificados e se o Tipo de Risco identificado poderia influenciar o Nível de Risco obtido por esses mesmos métodos.

Palavras chave: Análise de risco; Valoração de Risco; Perigo; Risco; Avaliação.

\section{RESUMEN}

La Evaluación del Riesgo: Comparación de distintos Métodos de Evaluación de Riesgos de la naturaleza semicuantitativa - Este artículo pretende presentar los resultados de un estudio realizado en el Master de Ergonomía en la Seguridad en el Trabajo, que se centró en el análisis comparativo de 10 métodos de evaluación de riesgos semicuantitativa. El propósito de este estudio fue investigar si la calificación de riesgo obtenidos en un 10 MERSqt fue idéntico para cada uno de los riesgos identificados y si el tipo de riesgo determinado podría afectar la calificación de riesgo obtenidos por estos métodos.

Palabras clave: Análisis de riesgos; Valoración de riesgos; Peligro; Riesgo; Evaluación .

\section{RESUMÉ}

Évaluation des Risques: Comparaison de Differentes Méthodes de Nature Semi-Quantitatif d'Evaluation des Risques - Cet article vise à présenter les résultats d'une étude menée au sein du Master de l'Ergonomie dans la Sécurité au Travail, qui a porté sur l'analyse comparative de 10 méthodes semi-quantitatives pour évaluer le risque. Cette étude visait à déterminer si la Niveau de risque obtenue par les 10 méthodes était identique pour chacun des risques identifiés précédemment et si le Type de Risque identifié pourraient influer sur la Niveau de Risque obtenue par cês méthodes.

Mots-clé: Analyse des risque; l'Évaluation des risque; Danger; Risque.

\section{ABSTRACT}

Risk Assessment: Comparison of Semi-Quantitative Risk Assessment Methods - This article intends to present the results of a study conducted within the Master of Ergonomics in Safety at Work, which focused on a comparative analysis between 10 methods. This study aimed to investigate whether the risk rating obtained by these methods was identical for each of the risks previously identified and if the type of risk identified could affect the risk rating obtained by these same methods.

Key words: Risk analysis; Risk evaluation; Hazard; Risk.

\footnotetext{
* O texto deste artigo corresponde à comunicação apresentada ao II Congresso Internacional de Riscos e VI Encontro Nacional, tendo sido submetido para revisão em 25-05-2010, tendo sido aceite para publicação em 26-06-2010.

Este artigo é parte integrante da Revista Territorium, n. ${ }^{\circ} 18,2011$, ® RIscos, ISBN: 0872- 8941.
} 


\section{Introdução}

A importância atribuída à Segurança e Saúde no Trabalho (SST) (Directiva 89/391/CEE do Conselho, de 12/06, e Lei $n .{ }^{\circ} 102 / 2009$, de 10 de Setembro) vem destacar o papel crucial que a Avaliação de Riscos assume em todo o processo, conferindo-lhe mesmo um lugar central nas abordagens preventivas. Na verdade, a preocupação de integrar a Avaliação de Risco na prevenção é referida no artigo $4^{\circ}$ da Convenção $n^{\circ} 155$ da OIT, de 22 de Junho de 1981 (OIT, 1981), onde se impõe aos Estados membros que a integram a implementação de uma política nacional que seja coerente, em matéria de SST e do Ambiente de trabalho, e que tenha por objectivo a prevenção dos acidentes de trabalho e dos perigos para a saúde dos trabalhadores, através da maior redução possível das causas dos riscos. Por seu turno, a Agência Europeia para a Segurança e Saúde no Trabalho (2008d), no seu documento Facts 81 (PT), refere que a avaliação de riscos constitui a base de uma gestão eficaz da SST e é fundamental para reduzir os acidentes de trabalho e as doenças profissionais. Se for bem realizada, esta avaliação pode melhorar a SST, bem como, de um modo geral, o desempenho das empresas.

Não obstante a obrigação legal associada à Avaliação de Risco, sabemos que é da responsabilidade de cada empresa e/ou serviço definir os processos de avaliação utilizados, já que em termos metodológicos não existem regras estabelecidas sobre a forma como esta deve ser realizada (F. Carvalho et al, 2007; F. CARValho et al, 2008).

A Comissão Europeia, num livro que publicou intitulado de "Guidance on risk assessment at work", corrobora esta ideia ao considerar que não existem regras estabelecidas sobre como levar a cabo uma avaliação de risco salientando, apenas, dois princípios que devem estar sempre presentes aquando da sua realização (European Commission, 1996):

- A avaliação de riscos deve ser estruturada para garantir que todos os perigos importantes, e respectivos riscos, não ficam esquecidos, mesmo aqueles associados às tarefas que são realizadas esporadicamente, ou fora do horário normal de trabalho;

- Quando um risco é identificado, deve-se começar por questionar se o risco é absolutamente necessário ou se pode ser eliminado pela abolição do perigo que o origina.

Segundo Ozog (2009), a Avaliação de Risco corresponde ao processo de identificação dos riscos de segurança e à determinação do custo efectivo na redução do mesmo. 0 mesmo autor salienta que, embora muitas organizações reconheçam a necessidade das avaliações de risco, muitas não têm as ferramentas, os recursos e a experiência para avaliar os riscos quantitativamente. Assim, estas organizações recorrem a métodos de avaliação de risco de natureza qualitativa (MAQl) ou de natureza semiquantitativa (MASqt), tais como as matrizes de risco.

L.A. Cox (2008) refere que as matrizes de risco são populares em múltiplas aplicações, nomeadamente na análise do risco no terrorismo, na gestão de projectos de construção de estradas, na construção de escritórios, nas mudanças de clima e na própria gestão de risco empresarial. Refere ainda que, apesar da sua utilização ser incentivada pelas próprias Normas Nacionais e Internacionais, entre várias organizações e consultores de risco, como são o caso da Military Standard 882C e AS/NZS 4360:1999, poucas pesquisas validam rigorosamente o seu desempenho na real melhoria das decisões de gestão do risco.

Embora as matrizes de risco sejam reconhecidamente fáceis de utilizar, podem originar falsos níveis de segurança, a menos que sejam adequadamente concebidas (OzoG, 2002).

Tendo presente que as avaliações de natureza semiquantitativa (MASqt) se tornam, na maior parte dos casos, as ferramentas disponíveis para levar a cabo as obrigações impostas pela legislação, já que são métodos generalistas e geralmente de fácil aplicação, não podemos descorar a lacuna existente na validação dos resultados das suas aplicações.

Poucos são os estudos que reflectem a preocupação de comparar os resultados das avaliações de risco aquando da utilização de diferentes métodos, em particular os métodos centrados nas matrizes de risco. Os poucos estudos que se conhecem (F. Carvalho, 2007 e J.C. Branco et al, 2007) reforçam a necessidade de se aprofundar o conhecimento científico neste domínio, para garantir a fiabilidade das avaliações de risco efectuadas.

Com este artigo pretendemos dar a conhecer o estudo realizado no âmbito do mestrado em Ergonomia na Segurança no Trabalho, no qual se realizou uma análise comparativa entre 10 métodos de avaliação de risco de natureza semi-quantitativa (MASqt), integrando um duplo objectivo:

- Por um lado, perceber se o Nível de Risco obtido pelos diferentes métodos utilizados é idêntico,

- E por outro lado, perceber se o Tipo de Risco em avaliação pode influenciar esses mesmos resultados.

De referir, que esta temática revelou-se, e continua a revelar-se, bastante pertinente e actual, facto evidenciado pelo lugar que ocupa nos congressos e encontros científicos na área da Segurança, Higiene e Saúde no Trabalho (SHST), de há uns anos a esta parte, sendo mesmo considerada uma das prioridades de intervenção estabelecidas na recentemente divulgada política de Segurança e Saúde da União Europeia, para o período 2007-2012 (Comissão das Comunidades Europeias, 2007). 


\section{Definição do Problema}

\section{Objectivos do estudo}

A aplicação de métodos de natureza semi-quantitativa de Avaliação de Risco constituiu o problema fulcral do estudo. A importância que estes métodos assumem na maioria das situações de trabalho levou-nos a pensar que era necessário obter informação mais precisa sobre o resultado que eles proporcionam. Estudos desta natureza têm-se revelado como sendo cada vez mais urgentes, sob pena de não se alcançarem os verdadeiros objectivos para os quais esses métodos são utilizados, em particular a:

- Identificação dos riscos que ultrapassam os níveis aceitáveis ou toleráveis;

- Fidedigna hierarquização dos riscos encontrados, de acordo com as prioridades de intervenção.

Em síntese, realizou-se um Estudo comparativo entre diferentes métodos de natureza Semi-quantitativa de Avaliação de Risco em Situação Real de Trabalho. Com o estudo referido procurou-se dar resposta às seguintes questões:

Q1 - "Será que o Nível de Risco obtido, pelos diferentes MASqt utilizados, é idêntico para cada um dos riscos/ consequências associadas?".

Q2 - "Será que o Tipo de Risco em avaliação influencia - Nível de Risco obtido pelos diferentes MASqt utilizados?".

As questões formuladas conduziram-nos à formulação das seguintes Hipóteses Nulas $\left(\mathrm{H}_{0}\right)$ :

$\mathrm{H}_{0}-1$ - Não existem diferenças significativas entre o Nível de Risco obtido pela aplicação dos diferentes MASqt.

$\mathbf{H}_{0}-\mathbf{2}$ - O Tipo de Risco em avaliação não influencia significativamente o Nível de Risco obtido pela aplicação dos diferentes MASqt.

Para testarmos as hipóteses atrás formuladas recorreuse à situação de trabalho de operadores de manutenção de uma empresa que operava no sector da Saúde e Bemestar, que por questões de confidencialidade não foi e não será aqui identificada.

\section{Metodologia}

Para o presente estudo foram aplicados 10 MASqt para estimar e valorar 150 riscos decorrentes da realização de 6 tarefas de manutenção. Este estudo foi desenvolvido segundo uma organização em 4 etapas.

\section{Etapas do estudo}

a) Caracterização da Situação de Trabalho (operadores de manutenção), a qual compreendeu:

Análise das características dos operadores (Condições Internas) - orientada para a caracterização dos sujeitos responsáveis por operações de manutenção (em termos de idade, género, antiguidade/experiência profissional, habilitações, formação/sensibilização no âmbito da SHST, queixas de saúde, entre outros).

Análise das características das tarefas (Condições Externas) - orientada para a identificação e caracterização das tarefas, quer em termos dos objectivos prescritos, quer em termos das condições de execução (em termos de características das instalações, das ferramentas, dos materiais, dos processos de trabalho, do ambiente físicoquímico, das condições organizacionais, bem como, dos equipamentos de protecção individual (EPI) disponibilizados).

b) Identificação dos Perigos, a qual compreendeu uma listagem dos perigos identificados e uma caracterização dos potenciais riscos e das eventuais consequências.

É de salientar que essas consequências foram apenas analisadas em termos dos danos a que os trabalhadores poderiam estar sujeitos aquando da exposição a esses mesmos perigos, deixando de lado qualquer avaliação, em termos de consequências sociais.

c) Estimativa do Risco, a qual implicou a caracterização do Risco, em termos das variáveis requeridas pelos 10 MASqt utilizados, a fim de quantificar a Magnitude do risco $(R)$.

d) Valoração do Risco, a qual permitiu a determinação da aceitabilidade do risco. Esta aceitabilidade decorreu da comparação da Magnitude do risco, encontrada na fase anterior, com o Índice de risco proposto pelos respectivos métodos utilizados. A valoração proporcionou a determinação do Nível de Risco, (a variável que estava em estudo) e, consequentemente, a hierarquização das necessidades/prioridades de intervenção.

\section{Amostras}

Para uma melhor compreensão do estudo, optámos por dividir a amostra nas sub-amostras que se seguem:

- Tarefas analisadas;

- Operadores;

- Riscos versus Consequências associadas 


\section{Tarefas analisadas}

Para a concretização deste estudo foram seleccionadas 6 tarefas de manutenção, de um total de 294 tarefas previstas no plano anual da empresa onde foi feito o estudo. A TABELA I sintetiza as tarefas que foram objecto de análise neste estudo, em termos de código, descrição e periodicidade com que são realizadas.

TABELA I - Síntese das tarefas analisadas.

\begin{tabular}{|c|c|c|}
\hline $\begin{array}{l}\text { Código } \\
\text { da } \\
\text { Tarefa }\end{array}$ & Descrição & Periodicidade \\
\hline $\mathrm{B}_{13}$ & $\begin{array}{l}\text { Lavagem dos filtros das UTA's (Unidades } \\
\text { de Tratamento de Ar) }\end{array}$ & Quinzenal \\
\hline$C_{7}$ & $\begin{array}{l}\text { Lavagem dos filtros (dos } \\
\text { Ventiloconvectores) e verificação das } \\
\text { pastilhas DEO (produto bactericida, } \\
\text { algicída e fungicida destinado a prevenir } \\
\text { e suprimir o crescimento da Legionella, } \\
\text { entre outros microorganismos, nos } \\
\text { sistemas de ar condicionado) }\end{array}$ & Quinzenal \\
\hline$I_{n}$ & $\begin{array}{c}\begin{array}{l}\text { Intervenções no Quadro eléctrico (QE) } \\
\text { - corresponde à realização de } 3 \text { sub- } \\
\text { tarefas: } \\
\mathrm{I}_{3} \text { - Execução de reapertos de } \\
\text { instalações eléctricas }\end{array} \\
\mathrm{I}_{4} \text { - Execução de medições de consumo } \\
\text { de energia } \\
\mathrm{I}_{5} \text { - Execução da limpeza do interior } \\
\text { do QE }\end{array}$ & Anual \\
\hline $\mathrm{J}_{4}$ & $\begin{array}{l}\text { Execução da trasfega de Químicos: } \\
\mathrm{NaOCl} \text { (Hipoclorito de Sódio em solução) } \\
\text { e } \\
\text { CTX-15 (preparação com Ácido Sulfúrico } \\
(38 \%) .\end{array}$ & Semanal \\
\hline $\mathrm{J}_{5}$ & $\begin{array}{c}\text { Execução da contra lavagem de } \\
\text { filtros e recarga com Diatomite (Pó } \\
\text { de cor branca, constituído por sílica } \\
\text { cristalizada). }\end{array}$ & Semanal \\
\hline $\mathrm{J}_{18}$ & $\begin{array}{l}\text { Lavagem do tanque de compensação do } \\
\text { Jacuzzi e da Piscina }\end{array}$ & Anual \\
\hline
\end{tabular}

Operadores

Neste campo referimo-nos aos indivíduos que foram objecto de observação durante a realização/análise das tarefas.

A análise das tarefas supra mencionadas contou com a colaboração de 5 operadores, todos do género masculino e com uma média de 29,4 anos de idade. Em termos de antiguidade na empresa, 40\% dos operadores desempenhava funções há menos de 1 ano e os restantes $60 \%$ entre 1 a 3 anos. No que se refere à experiência profissional em tarefas de manutenção, $60 \%$ ultrapassava os 6 anos de experiência, enquanto $40 \%$ situava-se no intervalo de $] 1$ a 3 anos].

Em termos de hábitos de vida, $60 \%$ era não fumador e os restantes $40 \%$ dividiam-se entre fumadores e ex-fumadores.

No período em que o estudo foi desenvolvido, apenas 40 $\%$ dos operadores faziam parte dos quadros da empresa em análise.
É de salientar que apenas $60 \%$ dos operadores tiveram formação específica para a realização das tarefas de manutenção executadas na empresa, sendo a tipologia dessa formação muito diversificada: por vezes era dada uma formação teórica complementada com formação prática de terreno, outras vezes a formação não passava de uma sensibilização passada no terreno, no acto da execução das tarefas.

No que se refere à formação/sensibilização no âmbito da SHST, $40 \%$ dos operadores referiram ter formação, $20 \%$ referiram ter tido uma sensibilização e os restantes $40 \%$ referiram não possuir qualquer conhecimento formalizado nessa matéria.

A formação/sensibilização referida no ponto anterior incidia nos seguintes campos:

- Utilização de Substâncias Perigosas;

- Trabalho com Electricidade;

- Movimentação Manual de Cargas;

- Equipamentos de Trabalho;

- Perigos associados às tarefas de manutenção;

- Sinalização de Segurança;

- Uso dos Meios de Combate a Incêndio;

- Espaços Confinados;

- Sensibilização para o uso de Equipamentos de Protecção Individual;

- Primeiros socorros.

Riscos versus Consequências associadas

Tendo presente os objectivos do estudo, a amostra utilizada para verificação das Hipóteses nulas apresentadas foi constituída pelos riscos, ou mais precisamente pelas potenciais consequências associadas aos riscos decorrentes dos perigos identificados na situação de trabalho em estudo. Deste modo, a nossa amostra foi condicionada pelas características das tarefas realizadas e pelas características dos operadores.

Desta forma, foram identificados 150 riscos, ou mais precisamente, 150 potenciais consequências associadas aos riscos decorrentes dos perigos identificados para a globalidade das tarefas analisadas na situação de trabalho em estudo, os quais constituíram a dimensão global da amostra para verificação da $\mathrm{H}_{0} 1$.

Para verificação da $\mathrm{H}_{0} 2$ houve a necessidade de agrupar os $150 \mathrm{riscos} /$ potenciais consequências associadas aos riscos decorrentes dos perigos identificados, por categorias de risco. Deste agrupamento resultaram 
15 categorias de risco (identificadas por $R_{n}$, onde $n$ corresponde ao $\mathrm{n}^{\circ}$ atribuído à categoria em avaliação). Dada a heterogeneidade do $\mathrm{n}^{\circ}$ de riscos por categoria e por forma a aumentar a potência dos resultados do teste, optámos por limitar o teste $\mathrm{da}_{\mathrm{H}} 2$ às categorias com $\mathrm{N} \geq 7$. A TABELA II apresenta a síntese das categorias de riscos que foram alvo deste teste.

TABELA II - Tipo/Categorias de riscos que foram integradas no teste de Friedman para testar $\mathrm{a}_{0} 2$ deste estudo.

\begin{tabular}{|c|c|c|}
\hline $\begin{array}{c}\text { Tipos } \\
\text { de Risco }\end{array}$ & Categoria de Riscos & N \\
\hline$R_{2}$ & Risco Eléctrico & 12 \\
\hline$R_{4}$ & Contacto directo com PQ & 36 \\
\hline$R_{5}$ & $\begin{array}{c}\text { Contacto directo com superfícies } \\
\text { cortantes }\end{array}$ & 9 \\
\hline$R_{7}$ & Contacto directo/Inalação de poeiras & 12 \\
\hline$R_{10}$ & Exposição ao Ruído & 12 \\
\hline$R_{11}$ & Queda a diferentes níveis & 8 \\
\hline$R_{13}$ & Risco de colisão & 7 \\
\hline$R_{14}$ & Sobresforço & 33 \\
\hline$R_{15}$ & $\begin{array}{c}\text { Tropeçamento/Escorregadela/Queda ao } \\
\text { mesmo nível }\end{array}$ & 8 \\
\hline
\end{tabular}

Recolha de dados

A recolha dos dados foi feita com recurso a diversas ferramentas/instrumentos de análise, de acordo com a especificidade das etapas em estudo.
Para uma melhor compreensão do que foi feito, apresentamos uma descrição das ferramentas/ instrumentos utilizada(os), por etapa, em paralelo com os objectivos e os procedimentos subjacentes à sua aplicação, sempre que se revele pertinente.

Na Caracterização da Situação de Trabalho ( $1^{\text {a }}$ etapa), a recolha dos dados foi feita a partir de observações livres e sistematizadas e recorreu a registo em vídeo, sonómetro, pesquisa documental e questionários desenvolvidos especificamente para esse fim.

Na TABELA III pode visualizar-se uma síntese dos objectivos e procedimentos associados a cada uma das ferramentas/ instrumentos de análise utilizadas ao longo desta etapa do estudo.

Na Identificação dos Perigos e associação dos potenciais riscos e respectivas consequências ( $2^{\mathrm{a}}$ etapa), a recolha dos dados foi realizada com auxílio de uma grelha de análise, em EXCEL, desenvolvida especificamente para esse fim (Fig. 1). Esta grelha, constituída por 8 campos (Componentes da Situação de Trabalho; Descrição da Situação ou da Tarefa; Circunstância perigosa; Perigo; Riscos associados; Consequência; Medidas de Segurança; Susceptibilidade Individual) objectivava reunir, num único ficheiro, toda a informação recolhida na etapa anterior e que se encontrava dispersa em vários documentos.

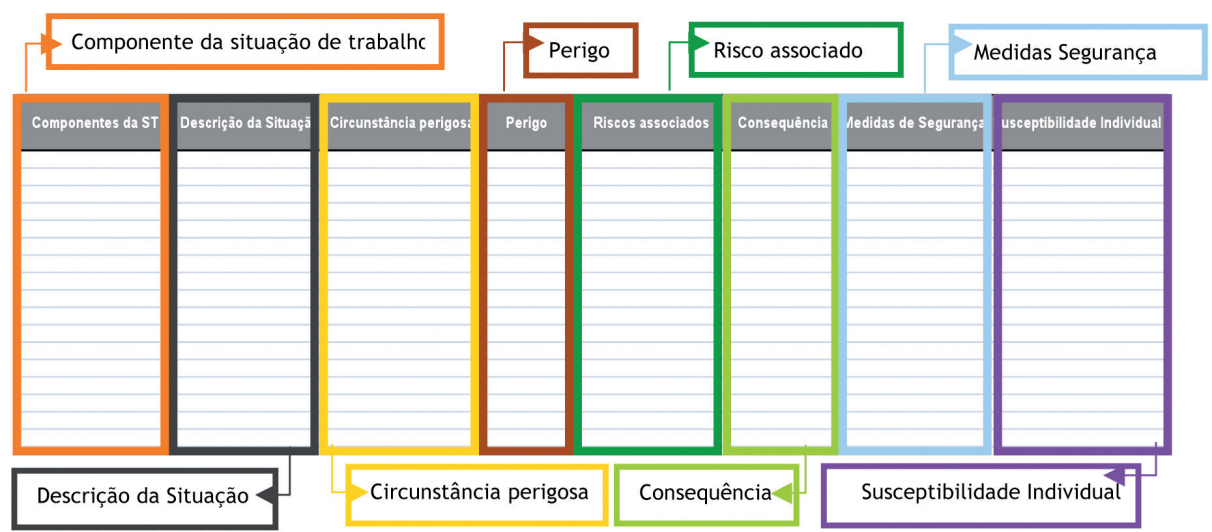

Fig. 1 - Exemplificação dos campos da grelha de análise, efectuada em EXCEL, para auxiliar a Etapa 2 do estudo. (Fonte: adaptado de F. Carvalho, 2007).

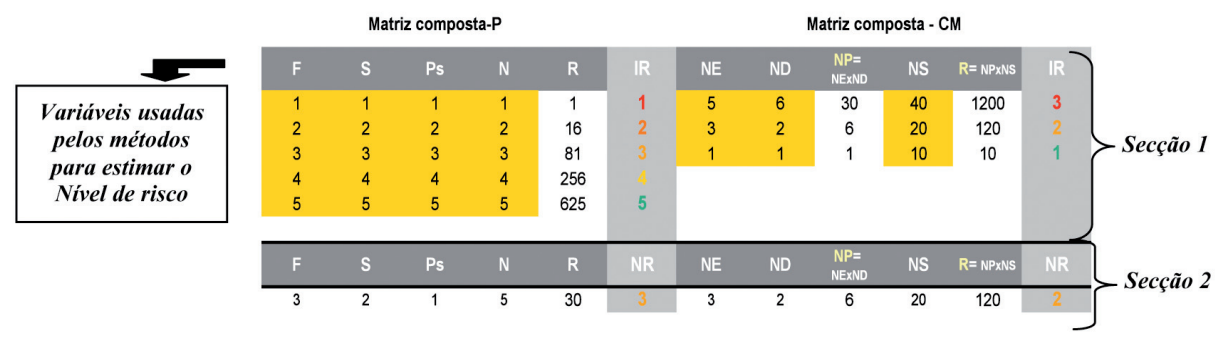

Legenda: (F) Frequência; (S) Severidade; (Ps) Procedimentos de segurança; (N) número de pessoas afectadas. (variáveis usadas no método de Matriz composta - P); (NE) Nível de exposição; (ND) Nível de deficiência; (NP) Nível de probabilidade; (NS) Nível de severidade. (variáveis usadas no método de Matriz composta - CM). (R) Magnitude do risco; (IR) Índice de risco.

Fig. 2 - Exemplificação da Grelha de análise, efectuada em EXCEL, para auxiliar a Etapa 3 e 4 do estudo. (Fonte: adaptado de F.CARVALHo, 2007). 
TABELA III - Síntese dos principais objectivos e das principias características/procedimentos subjacentes às ferramentas/instrumentos de análise utilizadas(os) ao longo do estudo.

Objectivos

instrumentos

:

Possibilitar uma caracterização adequada da situação de trabalho em estudo, nas suas duas principais componentes (condições internas e externas).

Recolher dados que possibilitassem contemplar a susceptibilidade individual na determinação da Probabilidade e/ou da Gravidade.

Recolher informação que, por não ser facilmente identificada pela simples observação, nos auxiliasse na caracterização da situação em estudo e, consequentemente, na identificação das circunstâncias perigosas.

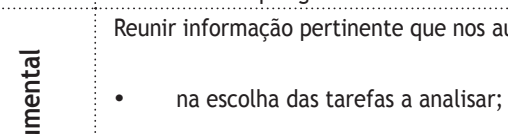

- na determinação dos conteúdos a contemplar no guia metodológico de observação (uma das ferramentas usadas);

- e na identificação dos perigos existentes.

Para possibilitar uma completa recolha de informação relativa aos vários elementos constituintes do posto de trabalho em estudo, desenvolveu-se uma grelha de análise, designada Guia metodológico de observação para caracterização da situação de trabalho, tornando-se a ferramenta auxiliar do método de observação.

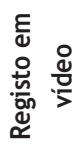

0 registo em vídeo foi a técnica escolhida para recolher imagens relativas à actividade de trabalho, aquando da realização das tarefas seleccionadas, por possibilitar uma caracterização mais criteriosa da mesma.

Para a caracterização da Situação de trabalho recorreu-se ainda ao uso de um Sonómetro digital da Roline - Digital Sound Level Meter RO - 1350 e respectivo calibrador acústico. 0 recurso a este equipamento objectivou minimizar a subjectividade associada à avaliação do parâmetro ambiental Ruído.
O questionário elaborado é composto por duas partes distintas (A e B) com objectivos igualmente distintos. Na parte A, procurou-se caracterizar a população, alvo de observação, através da determinação de indicadores como: idade, género, antiguidade, experiência profissional, formação/ sensibilização no âmbito da SHST, hábitos de vida, estado de saúde e doenças actuais. Na parte B, procurou-se recolher informação sobre a percepção do operador quanto às características das tarefas, em geral, e em termos das condições para a sua execução. A este nível foram contempladas questões para avaliar as características dos EPI (Equipamentos de Protecção Individual), das ferramentas de trabalho e do ambiente de trabalho.

Foram objecto de análise os seguintes documentos: legislação e normas aplicáveis, registos dos acidentes de trabalho ocorridos na empresa, plano das tarefas de manutenção, características dos EPI disponibilizados, fichas de segurança dos produtos químicos utilizados, características dos equipamentos alvo de manutenção, características das ferramentas/equipamentos utilizados, manual de SST da empresa e relatório de avaliações ambientais efectuadas.

Esta Grelha de análise está organizada por tópicos que procuram caracterizar os vários itens que determinam as principais condições de execução do trabalho:

A - Condições Organizacionais;

B - Equipamentos/Máquinas/Ferramentas

C - Movimentação Manual de Cargas

D - Outras acções vs Posturas

E - Produtos Químicos

F - Equipamentos de Protecção Individual (EPI)

G - Características das Instalações

$\mathrm{H}$ - Riscos Eléctricos

Foi utilizada uma câmara digital SONY Handycam, modelo DCR-DVD304E.

A recolha foi possível, graças ao consentimento prévio dos operadores envolvidos.

No processo de selecção de imagem procurámos captar todas as etapas subjacentes à realização das tarefas.

0 instrumento foi sujeito a uma verificação, no local de trabalho, antes e após cada série de medições, de acordo com o disposto no $\mathrm{n}^{\circ} 2$ do Anexo 1 do Decreto-lei n 182/2006 de 06/09 e tinha a calibração em dia. 
Na Estimativa do Risco ( $3^{\text {a }}$ etapa), para a caracterização do risco em termos Probabilidade e Gravidade foi criada uma extensão da Grelha de análise anterior, também em EXCEL. A Fig. 2 exemplifica a extensão da Grelha de análise, integrando apenas 2 métodos dos 10 utilizados.

A secção 1 corresponde à parte da Grelha onde os métodos se encontram descritos, ou seja, clicando em cima de cada uma das células, é-nos possível ter imediatamente a visualização de um comentário com o descritivo correspondente ao respectivo método. A secção 2 corresponde à parte da Grelha onde foi feita a análise propriamente dita. Na Fig.2, visualizar-se o exemplo de uma aplicação.

Na Valoração do Risco (4 $4^{\mathrm{a}}$ etapa), para a determinação da aceitabilidade do risco recorreu-se aos Índices de risco disponibilizados pelos 10 métodos aplicados na etapa anterior (estimativa do risco). Esta etapa foi realizada com o auxílio da Grelha abordada anteriormente.

\section{Métodos utilizados}

Foram utilizados 10 MASqt, dos quais:

- 5 integram-se numa categoria de métodos que recorrem ao uso de uma matriz simples, i.e, apenas fazem uso de duas variáveis para caracterizar a Magnitude do risco e onde a determinação do nível das escalas, das variáveis em jogo é da responsabilidade do analista;

- 5 integram-se numa categoria de métodos que recorre ao uso de uma matriz composta, i.e., fazem uso de três ou mais variáveis para caracterizar a Magnitude do risco e onde, a determinação do nível das escalas das variáveis em jogo nem sempre é da responsabilidade do analista, podendo resultar da combinação prévia entre outras das variáveis envolvidas, como é o caso do Nível de Probabilidade.

Para a realização deste estudo recorremos:

- por um lado, a métodos desenvolvidos por instituições/ organizações/empresas, do sector público e privado, gentilmente disponibilizadas, como foi o caso do Método de Matriz composta DGEMN (HIPólito, 2006), Método de Matriz composta-P e Método de Matriz composta-CM (sem divulgação da fonte por questões de confidencialidade).

- por outro lado, a métodos disponíveis na literatura tais como, Método de WTF (F. CABRAL et al, 2006; W.T.FINE, 1971), Método de matriz simples CRAM (CRAM, 2002), Método de matriz composta NTP330 (NTP 330, s/ data), Método de matriz simples $3 \times 3$ (M. HeRnández, et al, 1996) e, por fim, aos Método de matriz simples Somerville, Método de matriz simples $4 \times 4$, entre outros, apresentados por A.S.MIgUeL (2007).
Critérios usados na selecção dos métodos

A selecção dos métodos teve por base os seguintes critérios:

- Existência de, pelo menos, dois métodos com escalas idênticas de Índice de risco;

- Existência de, pelo menos, dois métodos, que se enquadrassem nas 2 categorias de métodos, acima referidos;

- Todos os métodos teriam que apresentar a variável Índice de risco expressa numa escala numérica;

- A Magnitude do risco teria que resultar de uma combinação, pré-estabelecida, entre as outras variáveis utilizadas pelo referido método (ex: A Probabilidade e Gravidade);

- Inexistência de obrigatoriedade na utilização de designações iguais para a identificação das variáveis implicadas na estimativa do risco. (ex: Probabilidade ou Frequência; Gravidade ou Severidade, apenas para citar alguns).

A TABELA IV apresenta uma síntese das principais características dos métodos utilizados.

\section{Critérios usados na aplicação dos métodos}

A aplicação dos métodos foi realizada sempre pelo mesmo analista para minimizar a subjectividade, eventualmente presente, aquando da atribuição dos níveis das escalas das variáveis postas em jogo por cada um dos métodos utilizados. Para além deste facto, o início de uma avaliação implicava a aplicação sequencial dos 10 métodos para garantir que os critérios se mantinham ao longo das várias metodologias em uso.

\section{Tratamento dos dados}

O tratamento da informação obtida, quer em termos descritivos quer em termos inferencial, foi feito com o auxílio do programa informático SPSS (Statistical Package for the Social Sciences - versão 15).

Considerando a natureza ordinal da variável em estudo, começámos por fazer uma análise descritiva dos dados, recorrendo a parâmetros de localização (moda, percentis), tabelas de frequência (simples e acumulada) e diagramas de barras.

O teste não paramétrico de FrIEDMAN foi a técnica estatística utilizada para testar as hipóteses formuladas: 
TABELA IV - Síntese das principais características dos métodos utilizados ao longo do estudo.

\begin{tabular}{|c|c|}
\hline Método & Principais características \\
\hline $\begin{array}{l}\text { Método de } \\
\text { matriz simples } \\
\text { Somerville }\end{array}$ & $\begin{array}{l}\text { É um método que recorre ao uso de uma matriz de análise de risco composta por duas escalas de três níveis, } \\
\text { para caracterizar a Gravidade }(\mathbf{G}) \text { e a Probabilidade }(\mathbf{P}) \text {; } \\
\text { Integra uma escala de Índice de risco para definir a prioridade de intervenção, igualmente com } 3 \text { níveis. }\end{array}$ \\
\hline $\begin{array}{l}\text { Método de } \\
\text { matriz simples } \\
(3 \times 3)\end{array}$ & $\begin{array}{l}\text { É um método que recorre ao uso de uma matriz em tudo semelhante à referida anteriormente (3X3), } \\
\text { simplesmente os descritores das escalas (G e P) assumem designações diferentes e a escala de Índice de } \\
\text { risco apresenta } 5 \text { níveis para definir a prioridade de intervenção. }\end{array}$ \\
\hline $\begin{array}{l}\text { Método de } \\
\text { matriz simples } \\
\text { CRAM }\end{array}$ & $\begin{array}{l}\text { É um método que, à semelhança dos anteriores, recorre a uma matriz que integra, para cada uma das } \\
\text { variáveis em estudo (G e P), uma escala de } 4 \text { níveis. Já no que se refere à escala de Índice de risco, para definir } \\
\text { a prioridade de intervenção, recorre a uma escala composta por } 3 \text { níveis. }\end{array}$ \\
\hline $\begin{array}{l}\text { Método de } \\
\text { matriz simples } \\
\quad(4 \times 4)\end{array}$ & $\begin{array}{l}\text { É um método que recorre ao uso de uma matriz com as mesmas características da anterior, já que também } \\
\text { recorre a duas escalas de } 4 \text { níveis, para caracterizar as variáveis } \mathbf{G} \text { e P. No entanto, para além dos descritores } \\
\text { assumirem designações diferentes, a escala de Índice de risco integra mais dois níveis, ou seja, apresenta um } \\
\text { total de } 5 \text { níveis de prioridade de intervenção. }\end{array}$ \\
\hline $\begin{array}{l}\text { Método de } \\
\text { matriz simples } \\
\quad(5 \times 4)\end{array}$ & $\begin{array}{l}\text { É um método que recorre ao uso de uma matriz composta por duas escalas de níveis diferentes. Assim, para } \\
\text { caracterizar a Frequência (F) (ou nível de Probabilidade de ocorrência dos eventos) é utilizada uma escala de } \\
5 \text { níveis, para caracterizar a Severidade (S) (também entendida como consequência ou Gravidade) é utilizada } \\
\text { uma escala de } 4 \text { níveis. A escala de Índice de risco integra } 4 \text { níveis de prioridade de intervenção. }\end{array}$ \\
\hline $\begin{array}{l}\text { Método } \\
\text { de matriz } \\
\text { composta-P }\end{array}$ & $\begin{array}{l}\text { É um método que recorre ao uso de uma matriz mais completa, comparativamente com as matrizes } \\
\text { abordadas anteriormente, já que integra mais duas variáveis (para além da Frequência (F) e da Severidade } \\
\text { (S)), sendo elas: os procedimentos e condições de segurança adoptados (Ps) e o no de pessoas afectadas (N). } \\
\text { Assim, cada uma das } 4 \text { variáveis (F, S, Ps e N) são analisadas com recurso a uma escala de } 5 \text { níveis. O produto } \\
\text { da classificação das } 4 \text { variáveis dá a Magnitude do risco. A escala varia entre } 1 \text { (muito mau) e } 625 \text { (muito } \\
\text { bom). Integra uma escala de Índice de risco com } 5 \text { níveis de prioridade de intervenção. }\end{array}$ \\
\hline $\begin{array}{l}\text { Método } \\
\text { de matriz } \\
\text { composta-CM }\end{array}$ & $\begin{array}{l}\text { É um método que, à semelhança do anterior, integra o conhecimento de } 4 \text { variáveis (Nível de exposição (NE), } \\
\text { Nível de deficiência (ND), Nível de probabilidade (NP) e Nível de Severidade (NS)). Cada uma destas variáveis } \\
\text { recorre a uma escala de } 3 \text { níveis. Neste método são utilizadas } 2 \text { matrizes de } 3 \text { X } 3 \text { que associam as variáveis } \\
\text { duas a duas (NE X ND e NP X NS). A escala de Índice de risco integra } 3 \text { níveis de prioridade de intervenção. }\end{array}$ \\
\hline $\begin{array}{l}\text { Método de } \\
\text { matriz composta } \\
\text { NTP } 330\end{array}$ & $\begin{array}{l}\text { É um método, em tudo semelhante ao anterior, já que requer o conhecimento de } 4 \text { variáveis, agora } \\
\text { designadas, por Nível de exposição (NE), Nível de deficiência (ND), Nível de probabilidade (NP) e Nível de } \\
\text { consequência (NC). Cada uma destas variáveis recorre, no entanto, a uma escala de } 4 \text { níveis. Neste método } \\
\text { são igualmente utilizadas } 2 \text { matrizes, de dimensões diferentes da anterior (4X4), as quais associam as } \\
\text { variáveis duas a duas (NE X ND e NP X NC). A escala de Índice de risco integra, agora, } 4 \text { níveis de prioridade } \\
\text { de intervenção. }\end{array}$ \\
\hline $\begin{array}{l}\text { Método de } \\
\text { matriz composta } \\
\text { DGEMN }\end{array}$ & $\begin{array}{l}\text { Método idêntico ao anterior, que recorre ao conhecimento de } 4 \text { variáveis, agora designadas por Níve } \\
\text { de exposição (NE), Nível dos procedimentos de segurança (NPS), Nível de Probabilidade (NP) e Nível de } \\
\text { Gravidade (NG). Cada uma destas variáveis recorre a uma escala de } 4 \text { níveis. Neste método são igualmente } \\
\text { utilizadas } 2 \text { matrizes de dimensão (4X4), as quais associam as variáveis duas a duas (NE X NPS e NP X NC). A } \\
\text { escala de Índice de risco integra, também, } 4 \text { níveis de prioridade de intervenção. }\end{array}$ \\
\hline $\begin{array}{l}\text { Método } \\
\text { William T. Fine } \\
\text { (WTF) }\end{array}$ & $\begin{array}{l}\text { É um método de Avaliação de Risco que recorre ao conhecimento de } 3 \text { variáveis, aqui designadas por Factor } \\
\text { consequência (Fc), Factor exposição (Fe) e Factor Probabilidade (Fp). Cada uma das } 3 \text { variáveis (Fc, Fe e Fp) é } \\
\text { analisada com recurso a uma escala de } 6 \text { níveis. O produto da classificação das } 4 \text { variáveis dá a Magnitude do } 0 \text { ) } \\
\text { risco ou GP (grau de perigosidade). A escala varia entre } 0.05 \text { (situação óptima) e } 10000 \text { (situação péssima). } \\
\text { Integra uma escala de Índice de risco com } 5 \text { níveis de prioridade de intervenção. }\end{array}$ \\
\hline
\end{tabular}

Fonte: (F. CARVALHO, 2007)

Em todos os testes foi considerado um nível de significância de 0,05.

Para a aplicação do teste de Friedman teve-se apenas em conta a comparação entre métodos que integravam Índices de risco com escalas idênticas. Desta forma, realizaram-se:

- 2 testes que envolveram 3 amostras emparelhadas (foi o caso dos 3 métodos que integram uma escala, para o Índice de risco, de 3 e 4 níveis, respectivamente);

- 1 teste que envolveu 4 amostras emparelhadas (foi o caso dos métodos que integravam uma escala de 5 níveis, para a variável - Índice de risco).

Para possibilitar uma análise directa dos dados, procedeu-se à conversão das escalas originais de alguns métodos, para que todos assumissem a mesma ordem, em termos da prioridade de intervenção a que cada nível corresponde. Assumiu-se que uma ordem crescente de níveis corresponderia a uma ordem decrescente de prioridade de intervenção. Esta opção levou-nos a uma alteração da ordem dos níveis de Índice de risco, inicialmente prevista para: Método de matriz simples Somerville; Método de matriz simples (3X3); Método de matriz simples (4x4); Método de matriz simples $(4 \times 5)$; Método de matriz composta - CM.

Esta alteração foi realizada com auxílio do SPSS.

Sempre que os resultados do teste de FRIEDMAN conduziram à rejeição da $\mathrm{H}_{0}$ foram efectuadas análises à posteriori para identificar os pares que conduziram a tais resultados. As análises à posteriori foram realizadas a partir da verificação da condição apresentada na equação (EQ.1): 


$$
\left|\bar{R}_{u}-\bar{R}_{v}\right| \geq Z_{a / k(k-1)} \sqrt{k(k+1) / 6 N}
$$

onde:

$\overline{\mathrm{R}}_{\mathrm{u}} \mathrm{e} \bar{R}_{v}=$ Mean rank obtido por cada k (para o respectivo par em avaliação);

$\mathrm{k}=\mathrm{n}^{\circ}$ de casos ou amostras;

$\mathrm{N}$ = dimensão das amostras;

$Z_{a / k(k-1)}=$ valor de abcissa de uma distribuição normal acima do qual se pode admitir que existe $a / k(k-1) \%$ da distribuição.

Paralelamente à realização do teste de Friedman e das análises à posteriori, este estudo contemplou a realização de análises aos descritores dos níveis das escalas de Índice de risco, procurando, neste sentido, verificar a consistência dos resultados apresentados.

Tendo presente que este estudo pretendeu avaliar o Risco individual, procurámos, ainda, identificar os métodos que se revelaram mais potentes do ponto de vista da protecção que conferem ao trabalhador. Para tal, analisaram-se os valores de Mean Rank sendo classificado como método mais protector aquele que assumia $1 .^{a}$ posição.

\section{Resultados e discussão}

Ao longo do trabalho, os resultados foram descritos em dois momentos:

1. ${ }^{\circ}$ momento - Análise descritiva do Nível de Risco obtido, por cada um dos métodos utilizados;

2. ${ }^{\circ}$ momento - Apresentação dos resultados dos testes às hipóteses nulas formuladas.

Tendo em conta os objectivos deste artigo, nomeadamente, a divulgação dos resultados do estudo centrado na análise comparativa entre $10 \mathrm{MASqt}$, optámos por, apenas, apresentar alguns dos resultados descritos no $2^{\circ}$ momento, i.e, correspondentes à apresentação dos resultados dos testes às hipóteses nulas formuladas.

\section{Resultados dos testes à $\mathrm{H}_{0} 1$}

Assumindo o agrupamento dos métodos em função do número de níveis da escala de Índice de risco, foram realizados 3 testes, dos quais:

a) Dois integraram 3 amostras (como foi o caso dos métodos de 3 e de 4 níveis de Índice de risco).

b) Um integrou 4 amostras (como foi o caso do grupo com 5 níveis de Índice de risco);

A TABELA $V$ sintetiza os resultados obtidos nos testes realizados para verificação da $H_{0} 1$. Da sua leitura pode evidenciar-se o seguinte: a) Em todos os casos se verificaram diferenças estatisticamente significativas, aquando da realização do teste não paramétrico de Friedman;

b) Apesar das diferenças encontradas, é possível constatar que:

- para o grupo de métodos com escala de Índice de risco de 3 níveis, para o par Método matriz simples - CRAM/Método matriz composta - CM não existem diferenças significativas, aquando da análise à posteriori;

- para o grupo de métodos com escala de Índice de risco de 5 níveis, para os pares Método matriz simples (3×3)/Método matriz composta - P, Método matriz simples $(4 \times 4) /$ Método matriz composta - $P$ e Método matriz simples $(4 \times 4) /$ Método de WTF não existem diferenças significativas, aquando da análise à posteriori.

c) Por outro lado, a análise efectuada aos descritores dos níveis de Índice de risco que assumiram maior frequência de casos veio revelar, de uma maneira geral, consistência nos resultados apresentados;

d) Uma análise aos métodos que assumiram a $1^{\mathrm{a}}$ posição, em relação ao valor de Mean rank, veio evidenciar que os métodos que se revelaram mais potentes, no que concerne à protecção que conferem ao trabalhador, são, por ordem crescente do $\mathrm{n}^{\circ}$ de níveis da escala de Índice de risco: 0 Método matriz simples Somerville; O Método matriz simples (4×5); O Método matriz simples $(3 \times 3)$.

\section{Resultados dos testes à $\mathrm{H}_{o} 2$}

Para testar a $\mathrm{H}_{0} 2$ seguimos a mesma lógica utilizada na $\mathrm{H}_{0} 1$, quer no tratamento dos dados, quer na apresentação dos resultados obtidos. Desta forma, foi realizado um total de 27 testes, i.e., foram realizados 3 testes (2 integrando 3 amostras, como foi o caso dos métodos de 3 e de 4 níveis de Índice de risco, e 1 integrando 4 amostras, como foi o caso dos métodos de 5 níveis de Índice de risco) para cada uma das 9 categorias de risco.

A TABELA VI sintetiza os resultados obtidos nos testes realizados para verificação da $\mathrm{H}_{0} 2$. Da sua leitura podemos salientar o seguinte:

a) Para o grupo de métodos com escala de Índice de risco de 3 níveis, apenas em 2 categorias de risco $\left(R_{5}\right.$ e $R_{10}$ ) não se encontraram diferenças estatisticamente significativas, revelando-se ser indiferente o método utilizado. No entanto, apenas um destes testes revelou consistência aquando da análise efectuada aos descritores dos níveis da escala de Índice de risco;

b) Para este grupo de métodos, a análise efectuada à posteriori veio evidenciar um conjunto de pares de 
TABELA $\mathrm{V}$ - Síntese dos resultados obtidos nos testes correspondentes à $\mathrm{H}_{0} 1$.
TABELA VI - Síntese dos resultados obtidos nos testes correspondentes à $\mathrm{H}_{0} 2$

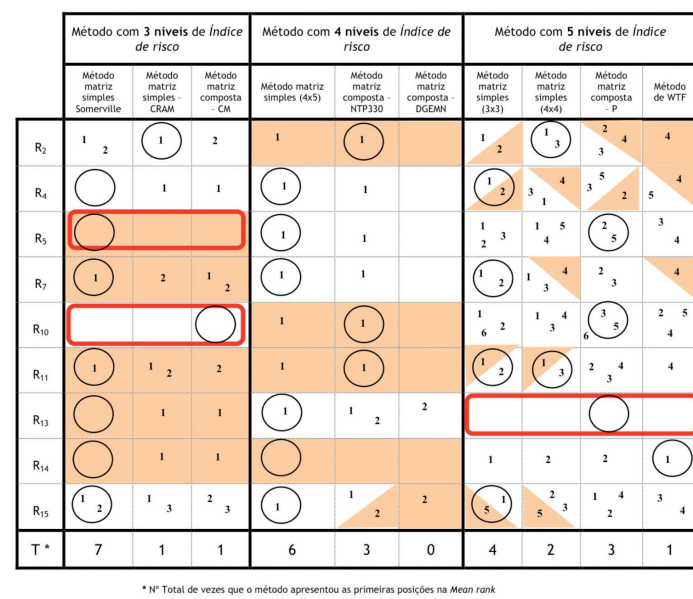

Fonte: (adaptado de F. CARVALHo, 2007)

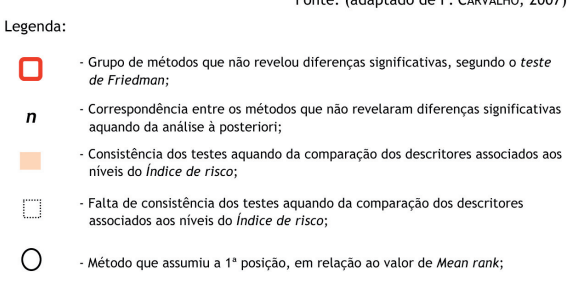

de diferenças significativas $\left(R_{13}\right)$, embora com baixa consistência;

h) As análises à posteriori realizadas aos restantes tipos de risco, vieram evidenciar a existência de vários pares de métodos que não apresentaram diferenças significativas. Apesar deste facto, poucos revelaram consistência aquando da análise efectuada aos descritores dos níveis de Índice de risco que assumiram maior frequência de casos.

i) Do ponto de vista da protecção que o método oferece ao trabalhador, as análises efectuadas à Mean rank vieram evidenciar que o resultado não era indiferente do método utilizado. Assim, constatou-se que o método de matriz simples $(3 \times 3)$ se revelou o mais potente, para a maioria das categorias de risco. A este nível gostaríamos de salientar, no entanto, que a disparidade dos resultados agora obtidos, comparativamente com os das análises realizadas para os métodos com escalas de Índice de risco de 3 e 4 níveis, vem reforçar a importância que o método pode ter na avaliação do risco.

\section{Conclusões}

$\mathrm{Na}$ generalidade dos casos, os resultados revelaram a existência de diferenças estatisticamente significativas entre os níveis de risco obtidos pelos diferentes MASqt (apenas 19\% apresentaram soluções equivalentes).

Para o grupo de métodos com escalas de Índice de risco com 5 níveis, apenas um teste revelou a inexistência 
Para a maioria das categorias de risco, o método a escolher deve ter em consideração o tipo de risco a avaliar.

Encontrou-se alguma unanimidade na potência revelada pelos métodos, no que se refere à protecção que conferem ao trabalhador, em particular, para os grupos de métodos com escalas de índice de risco de 3 e 4 níveis. A este nível destacaram-se:

- O Método de matriz simples Somerville (grupo de métodos com escala de Índice de risco de 3 níveis);

- O Método matriz simples $(4 \times 5)$ (grupo de métodos com escala de Índice de risco de 4 níveis).

Para o grupo de métodos com escala de Índice de risco de 5 níveis, não foi possível evidenciar com a mesma solidez, qual o método que se revela mais potente do ponto de vista da protecção que confere ao trabalhador. Esta situação veio evidenciar, no entanto, que para este grupo de métodos parece haver uma maior relação entre a protecção que o método confere ao trabalhador e a categoria de risco em avaliação.

Apesar das diferenças se terem revelado significativas na maioria dos testes realizados, a potência associada a esses resultados foi considerada limitada, já que, na nossa opinião, existe alguma incoerência entre os descritores associados a cada nível das escalas de Índice de risco disponibilizadas pelos métodos. Assim, parecenos lícito concluir que, independentemente da validade intrínseca de cada método, a sua escolha não deverá ser feita de forma aleatória.

A terminar, e tendo presente as vantagens associadas aos métodos de MASqt, consideramos que o problema investigado neste trabalho deve continuar a ser desenvolvido, no sentido de se realizarem análises mais profundas, no sentido de se encontrarem e definirem os critérios adequados à selecção dos métodos. A este nível salientamos o "escotoma" apresentado pela literatura, nessa matéria.

Os constrangimentos que sentimos, aquando do tratamento de dados, alertaram-nos para algumas alterações que deverão ser contempladas em análises futuras, nomeadamente, em relação às dimensões das escalas de Índice de risco e, sobretudo, em relação à equivalência entre os níveis das várias escalas.

Uma vez que o tipo de risco em avaliação parece influenciar os níveis de risco obtidos, pensamos que será interessante validar estes métodos de avaliação semi-quantitativa dos riscos (MASqt) em contextos diversificados, de forma a definir critérios adequados para a selecção mais fundamentada.

Por último, mas não menos importante, consideramos que uma análise comparativa inter e intra-analistas constitui uma matéria igualmente relevante no campo da investigação que é efectuada nesta área.

Estas orientações para pesquisas futuras podem, em nossa opinião, robustecer as Avaliações de Risco efectuadas e, consequentemente, o alcance dos objectivos subjacentes: a Prevenção da Saúde e Segurança das pessoas que trabalham.

\section{Referências bibliográficas:}

Agência Europeia para a Segurança e Saúde no Trabalho. (2008a). FACTS 76 (PT) - "Economia nacional e segurança e saúde no trabalho". Agência Europeia para a Segurança e Saúde no Trabalho.

Agência Europeia para a Segurança e Saúde no Trabalho. (2008b). FACTS 77 (PT) - "Vantagens para as empresas de uma boa segurança e saúde no trabalho". Agência Europeia para a Segurança e Saúde no Trabalho.

Agência Europeia para a Segurança e Saúde no Trabalho. (2008c). FACTS 80 (PT) - "Avaliação de riscos: funções e responsabilidades”. Agência Europeia para a Segurança e Saúde no Trabalho.

agência Europeia para a Segurança e Saúde no Trabalho. (2008d). Facts 81 (PT) - "Avaliação de riscos: a chave para locais de trabalho seguros e saudáveis”. Agência Europeia para a Segurança e Saúde no Trabalho.

Branco, J. C., Baptista, J. S., e Diogo, M. T. (2007). “Comparação da avaliação dos riscos por dois métodos correntemente utilizados na Industria Extractiva". In P. Arezes, J. Baptista, M. Barroso, A. Cunha, R. Melo, A. Miguel, et al. (Ed.), Colóquio Internacional sobre Segurança e Higiene Ocupacionais - SHO2008. Livro de Comunicações do SHO 2008, pp. 177-181. Guimarães: Sociedade Portuguesa de Segurança e Higiene Ocupacionais (SPOSHO) e Escola de Engenharia da Universidade do Minho.

Cabral, F., e Veiga, R. (Edits.). (2006). "Higiene, Segurança, Saúde e Prevenção de Acidentes de Trabalho" (20 ad., Vol. 1). VerLAG DASHÖVER.

Carvalho, F. (2007). "Estudo comparativo entre diferentes métodos de avaliação de Risco, em situação real de trabalho". Tese de Mestrado em Ergonomia na Segurança no Trabalho - FMHUTL. Cruz-Quebrada: (ref ${ }^{\mathrm{a}}$ não publicada).

Carvalho, F. e Melo, R. B. (2007). “Comparação entre Diferentes Métodos de Avaliação de Risco, em Situação Real de Trabalho". In C. Guedes Soares, A. P. Teixeira, e P. Antão, Riscos Públicos e Industriais (Vol. 2, pp. 853-868). Lisboa: Edições Salamandra. 
Carvalho, F., e Melo, R. B. (2008). “Comparação entre 10 métodos de Avaliação de Risco de natureza semi-quantitativa em tarefas de manutenção". In P. Arezes, J. Baptista, M. Barroso, A. Cunha, R. Melo, A. Miguel, et al. (Ed.), Colóquio Internacional sobre Segurança e Higiene Ocupacionais - SHO2008. Livro de Comunicações do SHO 2008, pp. 8387. Guimarães: Sociedade Portuguesa de Segurança e Higiene Ocupacionais (SPOSHO) e Escola de Engenharia da Universidade do Minho.

Comissão das Comunidades Europeias. (2007). "Melhorar a qualidade e a produtividade do trabalho: estratégia comunitária para a Saúde e Segurança no Trabalho 2007-2012”. COM (2007) 62 final, Comissão das Comunidades Europeias, Bruxelas.

Cox, L. A. (2008). “What's Wrong with Risk Matrices?”, Risk Analysis, 28 (2), 497-512.

CRAM. (2002). “Guide d'evaluation des risqué”. Disponível em: http://www.touraine.cci.fr/ formulaires/recherche/document_unique_ cram.pdf?PHPSESSID=a5c14df249aadff $5 f 8 f 7 c b$ 0645f835b4. Obtido em Fevereiro de 2006.

European Commission. (1996). "Guidance on risk assessment at work". Luxembourg: Office for official publications of the european communities.

FINE, W. (1971). "Mathematical evaluation for controlling hazards". Journal of safety research , 3 (4), 157-166.
Hernández, M., Fernández, E., Muñoz, G., e Prada, A. (1996). "Evaluatión de riesgos laborales". Madrid: Instituto Nacional de Seguridad e Higiene en el Trabajo.

Hipólito, J. (2006). “Modelo de gestão de riscos profissionais". Brochura- documento interno, DGEMN - Direcção Geral dos Edificios e Monumentos Nacionais, Lisboa.

Miguel, A. (2007). "Manual de Higiene e Segurança do Trabalho" (10 ed.). Porto: Porto Editora.

NTP 330. (s/ DATA). "Sistema simplificado de evaluación de riesgos de accidente". Disponivel em: http://www.mtas.es/insht/ntp/ntp_330.htm . Obtido em Fevereiro de 2006.

OIT. (1981). "Convenção n. ${ }^{\circ} 155$ de 22 de Junho de 1981 da OIT, sobre a Segurança, a Saúde dos Trabalhadores e o Ambiente de Trabalho". Genebra: Organização Internacional do Trabalho.

OIT. (2002). "Sistemas de Gestão da Segurança no Trabalho: directrizes práticas da OIT" $\left(1^{\text {a }}\right.$ ed., Vol. Informação Técnica). (M. Barroso, Trad.) Lisboa: IDICT - Instituto de Desenvolvimento e Inspecção das Condições de Trabalho.

Ozog. (2002). "Designing an Effective Risk Matrix”. Obtido em Abril de 2009, de http://www.iomosiac. com/docs/whitepapers/risk-ranking.pdf. 